\title{
SOME DIVISIBILITY PROPERTIES OF THE SUBGROUP COUNTING FUNCTION FOR FREE PRODUCTS
}

\author{
MICHAEL GRADY AND MORRIS NEWMAN
}

Dedicated to the memory of D. H. Lehmer

\begin{abstract}
Let $G$ be the free product of finitely many cyclic groups of prime order. Let $M_{n}$ denote the number of subgroups of $G$ of index $n$. Let $C_{p}$ denote the cyclic group of order $p$, and $C_{p}^{k}$ the free product of $k$ cyclic groups of order $p$. We show that $M_{n}$ is odd if $C_{2}^{4}$ occurs as a factor in the free product decomposition of $G$. We also show that if $C_{3}^{3}$ occurs as a factor in the free product decomposition of $G$ and if $C_{2}$ is either not present or occurs to an even power, then $M_{n} \equiv 0 \bmod 3$ if and only if $n \equiv 2 \bmod 4$. If, on the other hand, $C_{3}^{3}$ occurs as a factor, and $C_{2}$ also occurs as a factor, but to an odd power, then all the $M_{n}$ are $\equiv 1 \bmod 3$. Several conjectures are stated.
\end{abstract}

\section{INTRODUCTION}

A recurrence formula for the number of subgroups of a given index in a free group of finite rank was given by M. Hall [5]. This was generalized to the case of a free product of finitely many cyclic groups by I. M. S. Dey [2], and to the general case of an arbitrary group by $\mathrm{K}$. Wohlfahrt [9]. These numbers possess a wealth of fascinating arithmetic properties. For instance, the number of subgroups of index $n$ in the classical modular group is odd if and only if $n$ is of the form $2^{k}-3$ or $2\left(2^{k}-3\right)$ [6]. To cite another example, the number of subgroups of index $2 p-1$ in the Hecke group $H_{p}, p$ prime, is $2 p-1$ [4]. That these numbers are interesting is evident from Table 1 in the Appendix (included here as a representative example) which lists them for $H_{41}$, for all indices $\leq 100$.

In this paper we prove a number of congruence properties, and state several conjectures.

$C_{p}$ will denote the cyclic group of order $p . C_{p}^{k}$ will stand for the free product $C_{p} * C_{p} * \cdots * C_{p}$ of $k$ copies of $C_{p}$. Then the classical modular group is $C_{2} * C_{3}$, and $H_{p}$ is $C_{2} * C_{p}$.

\section{The Hall and Dey formulas}

Let $M_{n}$ denote the number of subgroups of index $n$ in some specified group $G$. Then, if $G$ is a free group of rank $r, M_{1}=1$ and

Received July 16, 1990; revised October 2, 1990.

1991 Mathematics Subject Classification. Primary 11B50, 20 E06.

(C) 1992 American Mathematical Society $0025-5718 / 92 \$ 1.00+\$ .25$ per page 


$$
M_{n}=n(n !)^{r-1}-\sum_{i=1}^{n-1}(n-i) !^{r-1} M_{i}, \quad n \geq 2 .
$$

If $G=C_{p_{1}} * C_{p_{2}} * \cdots * C_{p_{k}}$, then $M_{1}=1$ and

$$
M_{n}=\frac{h(n)}{(n-1) !}-\sum_{i=1}^{n-1} \frac{h(n-i)}{(n-i) !} M_{i}, \quad n \geq 2,
$$

where

$$
h(n)=\tau_{p_{1}}(n) \tau_{p_{2}}(n) \cdots \tau_{p_{k}}(n),
$$

and $\tau_{p_{i}}(n)$ is the number of homomorphisms of $C_{p_{i}}$ into the symmetric group $S_{n}$.

Both (1) and (2) have an equivalent formulation in terms of generating functions.

For Dey's formula (2), let

$$
g=\sum_{n=0}^{\infty} M_{n+1} x^{n}, \quad f=\sum_{n=0}^{\infty} \frac{h(n)}{n !} x^{n},
$$

where $h(0)$ is defined to be 1 . Then

$$
g=\frac{f^{\prime}}{f}
$$

This simple expression is useful in a variety of counting arguments (see [3, 4], for example).

\section{CONGRUENCE PROPERTIES, AND THE THEOREMS}

Parity phenomena are rather obvious for free groups of finite rank. In fact, all the $M_{n}$ are odd, in this case. This follows trivially by induction from Hall's formula (1), when considered modulo 2 . In fact, the behavior of these numbers can be determined (in principle) for any modulus, since (1) then becomes a linear recurrence of fixed length with constant coefficients. For example, choosing 5 as the modulus and the rank as 2 , the recurrence becomes

$$
M_{n} \equiv 4 M_{n-1}+3 M_{n-2}+4 M_{n-3}+M_{n-4}, \quad n \geq 5,
$$

with initial values

$$
M_{1} \equiv 1, \quad M_{2} \equiv 3, \quad M_{3} \equiv 3, \quad M_{4} \equiv 1 .
$$

The period of this sequence is 62 , which determines the behavior of the indices modulo 5 completely. For example, this implies that $M_{n}$ is divisible by 5 if and only if

$$
\begin{aligned}
n \equiv & 9,12,19,24,33,39,41,42, \\
& 45,47,49,52,58,59,60 \bmod 62 .
\end{aligned}
$$

In the same way, it is easy to show that for a free group of rank $p$, where $p$ is a prime, $M_{n}$ satisfies $M_{n} \equiv 1 \bmod p$ for all $n$.

The group-theoretic significance of results of this type is rather obscure. 
Establishing congruence patterns for free products tends to be more challenging, since equation (2) is less convenient than (1) for that purpose. Instead of appealing to (2) directly, W. W. Stothers in [6] derives a formula for $M_{n}$ for the modular group via coset diagrams. This formula is then used to prove the parity result mentioned above. These methods were extended in [8] and [7].

An interesting alternative approach was given by C. Godsil, W. Imrich, and R. Razen in [3]. They obtain a recurrence formula for $\frac{\tau_{2}(n) \tau_{3}(n)}{n !}$, and from this a recurrence for $M_{n}$ via equations (3) and (4). Congruence properties are then deduced from this recurrence. The same reference mentions that the number of free subgroups of index $n$ in $\mathrm{SL}_{2}(\mathbb{Z})$ is always even. This follows from a formula given by W. Imrich in [8].

T. Müller has found that $\mathrm{SL}_{2}(\mathbb{Z})$ exhibits the same parity pattern as the modular group [10], and more generally that similar patterns hold for a variety of free products of finite groups for which the amalgamated subgroup has odd cardinality.

In what follows we will show that Dey's formula reduces to a linear recurrence modulo $p$ when the factors are appropriately chosen. This enables us to prove the following theorems:

Theorem 1. Suppose that $C_{2}^{4}$ occurs as a factor in the free product decomposition of $G$. Then $M_{n}$ is odd for all $n \geq 1$.

Theorem 2. Suppose that $C_{3}^{3}$ occurs as a factor in the free product decomposition of $G$. If $C_{2}$ does not occur as a factor, or enters to an even power, then $M_{n} \equiv 0$ mod 3 if and only if $n \equiv 2 \bmod 4$. If, on the other hand, $C_{2}$ enters to an odd power, then $M_{n} \equiv 1 \bmod 3$ for all $n$, so that $M_{n}$ is never divisible by 3 in this case.

The proofs require a number of lemmas.

Lemma 1. The number $\tau_{2}(n)^{4}$ contains a higher power of 2 in its prime power factorization than does $n$ !, for all $n>1$.

Proof. Let $2^{r_{n}}$ be the exact power of 2 dividing $n$ !, and let $2^{k}$ be the largest power of 2 less than or equal to $n$, so that

$$
r_{n}=\left[\frac{n}{2}\right]+\left[\frac{n}{2^{2}}\right]+\cdots+\left[\frac{n}{2^{k}}\right]
$$

Clearly, if $n=2^{k}$, then $r_{n}=2^{k}-1=n-1$, but otherwise, $r_{n}<n-1$.

From S. Chowla et al. [1] we have $\tau_{2}(n) \equiv 0 \bmod 2^{s}$ for any $s$ such that $s \leq(n+2) / 4$. Thus, if $n \equiv 2 \bmod 4$, we may choose $s=(n+2) / 4$ and deduce that $\tau_{2}(n)^{4} \equiv 0 \bmod 2^{n+2}$. Since $n+2>r_{j}$ for $j=n, n+1, n+2$, or $n+3$ (even in the case $n+2=2^{k}$ ), the result follows.

We can now prove Theorem 1 . We have $M_{1}=1$, and by Lemma 1 and formula (2),

$$
M_{n} \equiv M_{n-1} \bmod 2, \quad n>1 .
$$

The result now follows trivially, by induction.

The next lemma is considerably more difficult to prove.

Lemma 2. If $n \geq 9 s-3$, then $\tau_{3}(n) \equiv 0 \bmod 3^{2 s}$. 
Proof. We shall prove inductively the compound proposition: If $n \geq 9 s-3$, then $\tau_{3}(n) \equiv 0 \bmod 3^{2 s}$, and for $n \equiv 1 \bmod 3$,

$$
\tau_{3}(n) \equiv \tau_{3}(n-1) \quad \bmod 3^{2 s+1} .
$$

This proposition may be verified by direct computation for $s=1,2$. For brevity, let $T_{n}$ now denote $\tau_{3}(n)$. Iteration of the fundamental recursion

$$
T_{n}=T_{n-1}+(n-1)(n-2) T_{n-3}
$$

gives

$$
T_{n}=x T_{n-7}+y T_{n-8}+z T_{n-9},
$$

where

$$
\begin{aligned}
& x=x(n)=3 n^{4}-42 n^{3}+210 n^{2}-441 n+351, \\
& y=y(n)=3 n^{4}-54 n^{3}+342 n^{2}-903 n+882, \\
& z=z(n)=n^{6}-27 n^{5}+289 n^{4}-1569 n^{3}+4579 n^{2}-6927 n+4536 .
\end{aligned}
$$

Suppose that $n \geq 9(s+1)-3$. Then by the induction hypothesis, $T_{n-7}, T_{n-8}$, and $T_{n-9}$ are all divisible by $3^{2 s}$; and we wish to show that $T_{n} \equiv 0 \bmod 3^{2 s+2}$. So let $k=2 s, T_{n-7}=3^{k} a, T_{n-8}=3^{k} b, T_{n-9}=3^{k} c$. We must show that

$$
x a+y b+z c \equiv 0 \bmod 9 .
$$

Working modulo 9, we have

$$
\begin{aligned}
& x \equiv 3 n^{4}+3 n^{3}+3 n^{2}, \\
& y \equiv 3 n^{4}-3 n, \\
& z \equiv n^{6}+n^{4}-3 n^{3}-2 n^{2}+3 n .
\end{aligned}
$$

It is readily verified that $z \equiv 0 \bmod 9$ for all $n$ and that $x \equiv y \equiv 0 \bmod 9$ unless $n \equiv 2 \bmod 3$. So let $n \equiv 2 \bmod 3$. Then $T_{n} \equiv 3 a+6 b \equiv 0 \bmod 9$ requires $a \equiv b \bmod 3$; i.e., $T_{n-7} \equiv T_{n-8} \bmod 3^{k+1}$. By virtue of (6), this last congruence becomes

$$
\begin{aligned}
& x_{1} T_{n-14}+y_{1} T_{n-15}+z_{1} T_{n-16} \\
& \quad \equiv x_{2} T_{n-15}+y_{2} T_{n-16}+z_{2} T_{n-17} \quad \bmod 3^{k+1},
\end{aligned}
$$

where we do not bother to write down the coefficients explicitly, but note that $x_{1}, y_{1}, z_{1}$ are to be evaluated at $n-7 \equiv 1 \bmod 3$, and $x_{2}, y_{2}, z_{2}$ are to be evaluated at $n-8 \equiv 0 \bmod 3$. By the induction hypothesis, $T_{n-14}, \ldots, T_{n-17}$ are all divisible by $3^{k-2}$. It is a simple matter to show that $x(m) \equiv 0 \bmod 27$ when $m \equiv 0$ or $1 \bmod 3$, and that $y(m) \equiv 0 \bmod 27$ when $m \equiv 1 \bmod 3$. Thus, it remains to prove that

$$
\left(z_{1}-y_{2}\right) T_{n-16} \equiv z_{2} T_{n-17} \bmod 3^{k+1} \text {. }
$$

Let $T_{n-16}=3^{k-2} a, T_{n-17}=3^{k-2} b$. By the induction hypothesis, $T_{n-16} \equiv$ $T_{n-17} \bmod 3^{k-1}$, which implies that $a \equiv b \bmod 3$. But $z_{2} \equiv 0 \bmod 9$, and

$$
\begin{aligned}
& z_{1}(n+1)-y_{2}(n)=n^{6}-21 n^{5}+166 n^{4}-609 n^{3}+1009 n^{2}-546 n \\
& \quad \equiv n^{6}-3 n^{5}+4 n^{4}-6 n^{3}+n^{2}-6 n \bmod 9,
\end{aligned}
$$

which is clearly $\equiv 0 \bmod 9$ when $n \equiv 0 \bmod 3$. This completes the proof.

We use this lemma to prove 
Lemma 3. The number $\tau_{3}(n)^{3}$ contains a higher power of 3 in its prime power factorization than does $n$ !, for all $n>2$.

Proof. Let $3^{r_{n}}$ be the exact power of 3 dividing $n$ !, and let $3^{k}$ be the largest power of 3 less than or equal to $n$, so that

$$
r_{n}=\left[\frac{n}{3}\right]+\left[\frac{n}{3^{2}}\right]+\cdots+\left[\frac{n}{3^{k}}\right]
$$

Then $r_{n} \leq(n-1) / 2$, with equality only when $n=3^{k}$. Suppose that $n \equiv 6$ $\bmod 9$, and $s=(n+3) / 9$. By Lemma 2 ,

$$
\tau_{3}(n)^{3} \equiv 0 \bmod 3^{(6 n+18) / 9} \text {. }
$$

But $(6 n+18) / 9>r_{j}$ for $j=n, n+1, \ldots, n+8$ and $n>9$. Direct computation verifies the lemma for $n=1,2, \ldots, 9$. This completes the proof.

We can now prove Theorem 2. Suppose, first, that $C_{2}$ does not occur as a factor in the free product decomposition of $G$. Then if $p$ is a prime $>2$ such that $C_{p}$ does occur as a factor, the fact that $\tau_{p}(1)=\tau_{p}(2)=1$ together with Lemma 3 implies that

$$
M_{n} \equiv 2 M_{n-1}+M_{n-2} \bmod 3, \quad n>2,
$$

with initial values $M_{1} \equiv 1 \bmod 3, M_{2} \equiv 0 \bmod 3$. This linear recurring sequence has period 8 and produces the values

$$
1,0,1,2,2,0,1,1,0, \ldots
$$

so that $M_{n} \equiv 0 \bmod 3$ if and only if $n \equiv 2$ or $6 \bmod 8$; or what is the same thing, if and only if $n \equiv 2 \bmod 4$.

Now suppose that $C_{2}^{k}$ does occur as a factor in the free product decomposition of $G$. Formula (7) is affected by this and becomes instead

$$
M_{n} \equiv 2 M_{n-1}+2^{k} M_{n-2} \bmod 3, \quad n>2 \text {, }
$$

with initial values $M_{1} \equiv 1 \bmod 3, M_{2} \equiv 2^{k}-1 \bmod 3$. If $k$ is even, the recurrence is unaffected and the desired conclusion holds. If $k$ is odd, however, we find in this case that all the $M_{n}$ satisfy $M_{n} \equiv 1 \bmod 3$. This completes the proof.

\section{Conjectures}

We conclude this paper by listing some plausible conjectures, which are backed up by some massive calculations.

Conjecture 1. If $C_{2}^{2}$ occurs as a factor in the free product decomposition of $G$, then $M_{n}$ is odd for all $n$.

Conjecture 2. Let $G=C_{p}^{2} * C_{p_{1}} * \cdots * C_{p_{k}}$, where $p_{i} \geq p$ for all $i$. Then the residue of $M_{n}$ modulo $p$ for $G$ is the same as for the group $C_{p}^{2}$, and this is given recursively by

$$
M_{n} \equiv-\sum_{i=1}^{p-1} \frac{1}{(p-i) !} M_{n-p+i} \bmod p, \quad n>p-1 .
$$

Thus, for example, the group $C_{2}^{2} * C_{3}^{2}$ has all $M_{n}$ odd, and $M_{n} \equiv 0 \bmod 3$ if and only if $n \equiv 2 \bmod 4$. 
Conjecture 3. Let $p_{1}, p_{2}$ be primes, and suppose that $p_{1} \equiv 1 \bmod q$, where $q$ is a prime. Let $G=C_{p_{1}} * C_{p_{2}}$. Then $M_{n} \equiv 0 \bmod q$ for all $n$ such that $n \not \equiv 1$ $\bmod q, n \not \equiv p_{2} \bmod q$.

\section{APPENDIX}

\section{TABLE 1}

The function $M n$ is the number of subgroups of index $n$ of the Hecke group $H(41)$, the free product of a cyclic group of order 2 and a cyclic group of order $41 \cdot M n$ is 1 for $n=1,2$, and is 0 for $n=3,4, \ldots, 40$.

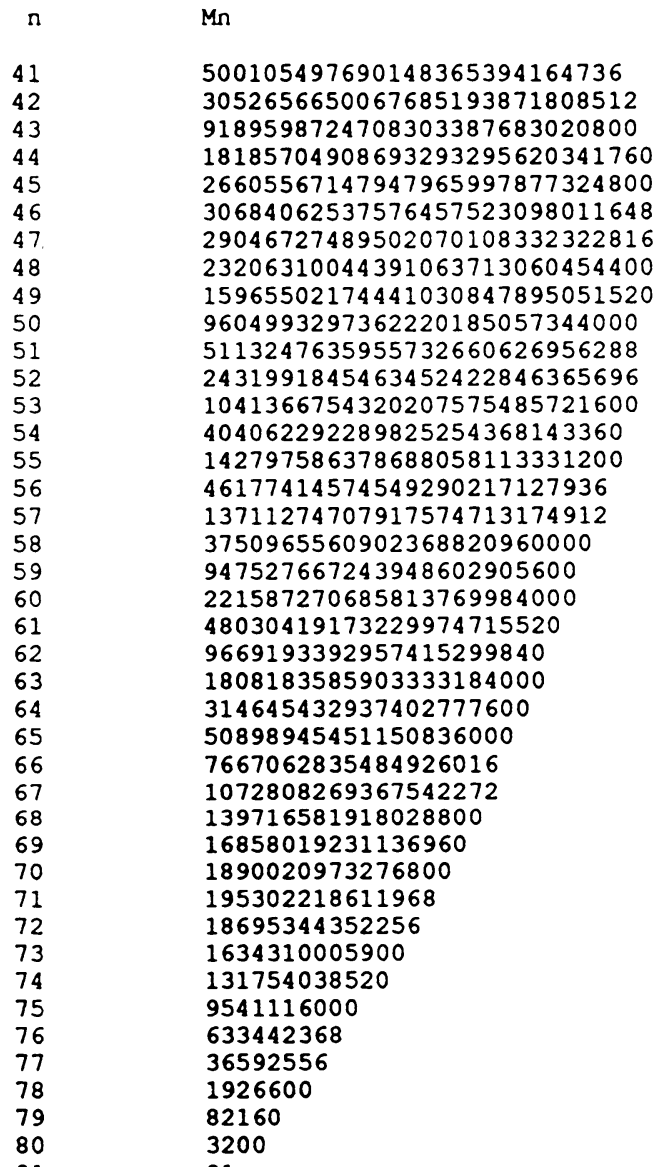

81

542443923271892169723911087435440323085660066294811429302173698 4719313572641000369499585833970161707226694837279245830706954240 20394558391041910084364370737774577370079277452472143590761431040 58366814703260040549315958464995086264884454105320124609304985600 124437583317103197230808365069305154113452274534860785787590410240 210796692105067676310277499630992571656008299258349131115440111616 295525242459188893735690544829860554750241649011037383009721384960 352647113397445563325134294321169800442721432180072037816876400640 365609018450742293149063258637877217626417465023199778515517440000 334520997815810176042582916043167517149758918629609968362271539200 273472417847956512216462005531448828091591579380023778382918451200 201748067392705380123380252578618239256892196807820427837429514240 135416412488552276293181468005171653646550381107918875734963650560 83268423490801113887128378022781441560556679176648989993861120000 47181037122994202406619926137577333046941929607442593668608819200 24757449861030329499521590344703990734983602083960099275373281280 12083167101655322150101011754513036933215073150832541218916597760 5506054103469592601355901745089186309104848528304523538532925440 2350378790138361059233610111228341650466688830421212035809280000 


\section{ACKNOWLEDGMENT}

The authors are indebted to the referee for several helpful comments and for drawing our attention to reference [10].

\section{BIBLIOGRAPHY}

1. S. Chowla, I. N. Herstein, and K. Moore, On recursions connected with symmetric groups. I, Canad. J. Math 3 (1951), 328-334.

2. I. M. S. Dey, Schreier systems in free products, Proc. Glasgow Math. Assoc. 7 (1965), 61-79.

3. C. Godsil, W. Imrich, and R. Razen, On the number of subgroups of given index in the modular group, Monatsh. Math. 87 (1979), 273-280.

4. M. Grady and M. Newman, Counting subgroups of given index in Hecke groups, Contemp. Math., Amer. Math. Soc. (to appear).

5. M. Hall, Subgroups of finite index in free groups, Canad. J. Math. 1 (1949), 187-190.

6. W. W. Stothers, The numbers of subgroups of given index in the modular group, Proc. Roy. Soc. Edinburgh Sect. A 78 (1977), 105-112.

7. __ Subgroups of finite index in a free product with amalgamated subgroup, Math. Comp. 36 (1981), 653-662.

8. W. Imrich, On the number of subgroups of a given index in $S L_{2}(\mathbb{Z})$, Arch. Math. 31 (1978), 224-231.

9. K. Wohlfahrt, Über einen Satz von Dey und die Modulgruppe, Arch. Math. 29 (1977), 455-457.

10. T. Müller, Kombinatorische Aspekte endlich erzeugter virtuell freier Gruppen, Dissertation, Johann Wolfgang Goethe-Universität, 1989.

Department of Mathematics, University of California, Santa Barbara, California 93106

E-mail address: newman\%henri@hub.ucsb.edu 